\title{
Gestión de recursos hídricos: una revisión internacional de literatura
}

Water resources management: an international literature review

Gestão de recursos hídricos: uma revisão da literatura internacional

\author{
Jhonny Gárate Ríos \\ jgarater@ucvvirtual.edu.pe \\ Universidad Cesar Vallejo (UCV) - Perú \\ http://orcid.org/0000-0002-3062-6106
}

\author{
Gabriela del Pilar Palomino Alvarado \\ dpalominoal@ucvvirtual.edu.pe \\ Universidad Cesar Vallejo (UCV) - Perú \\ http://orcid.org/0000-0002-2126-2769
}

Tony Venancio Pereyra Gonzales
pereyragt@ucvvirtual.edu.pe
Universidad Cesar Vallejo (UCV) - Perú
http://orcid.org/0000-0003-3833-2126

Froy Torres Delgado

ftorres@unsm.edu.pe

Universidad Cesar Vallejo (UCV) - Perú http://orcid.org/0000-0002-1056-3670

RESUMEN

La investigación tuvo como objetivo identificar y examinar sistemáticamente la evidencia científica disponible sobre la gestión de los recursos hídricos en las cuencas de nuestro planeta, disponible en la base de datos EBSCO, Scopus, Science Direct, SciELO, ProQuest y Redalyc en inglés, portugués y español de los últimos 5 años. Método: Se ingresó a las bases de datos, realizándose la búsqueda del término "gestión de recursos hídricos", descartándose artículos que no sean arbitradas y académicas y hayan sido publicados antes del año 2016. Como resultado de ello, se evidenció que existe la urgente necesidad de nuevos paradigmas para mejorar la gestión de recursos hídricos y para ello se debe consolidar una autoridad descentralizada sobre el agua. La investigación concluye que, los tres pilares; económico, social y ambiental, deben estar articulados para lograr la consolidación de la Gestión de los Recursos Hídricos, además; se puede afirmar que existen distintos sectores que supervisan la gestión de los recursos hídricos con regulaciones compartidas y responsabilidades superpuestas, incluso en conflicto; lo cual consolida la burocracia hídrica del estado. A esto se suma el crecimiento demográfico que genera un fuerte impacto sobre el cambio climático y acrecienta la escasez del agua.

Palabras claves: Gestión Integrada. Recursos Hídricos. Gobernanza. Água.

\begin{abstract}
The research aimed at systematically identifying and examining the available scientific evidence on the management of water resources in the basins of our planet, available in the EBSCO, Scopus, Science Direct, SciELO, ProQuest and Redalyc databases in English, Portuguese and Spanish for the last 5 years. Method: The databases were entered, searching for the term "water resources management", discarding articles that were not refereed and academic and had been published before 2016. As a result, it was evidenced that there is an urgent need of new paradigms to improve the management of water resources and for this, a decentralized authority on water must be consolidated. The research concludes that, the three pillars; economic, social and environmental must be articulated to achieve the consolidation of the Management of Water Resources, in addition; It can be affirmed that there are different sectors that supervise the
\end{abstract}


management of water resources with shared regulations and overlapping responsibilities, even in conflict; which consolidates the state's water bureaucracy.

Keywords: Integrated Management. Water Resources. Governance. Water.

\section{RESUMO}

O objetivo da pesquisa teve como objetivo identificar e examinar sistematicamente as evidências científicas disponíveis sobre a gestão dos recursos hídricos nas bacias do nosso planeta, disponíveis nas bases de dados EBSCO, Scopus, Science Direct, SciELO, ProQuest e Redalyc em inglês, português e espanhol dos últimos 5 anos. Método: Foram inseridos os bancos de dados, realizando-se procura do termo "gestão de recursos hídricos", descartando-se os artigos que não foram referenciados e acadêmicos e que haviam sido publicados antes do ano de 2016. Como resultado, evidenciou-se que há uma urgência de novos paradigmas para melhorar a gestão dos recursos hídricos e, para isso, deve-se consolidar uma autoridade descentralizada sobre a água. A pesquisa conclui que, os três pilares; econômica, social e ambiental, devem estar articuladas para alcançar a consolidação da Gestão de Recursos Hídricos, além disso; pode-se dizer que existem diversos setores que fiscalizam a gestão dos recursos hídricos com regulações compartilhadas e responsabilidades sobrepostas, mesmo em caso de conflito; o qual consolida a burocracia hídrica do estado. Soma-se a isso o crescimento demográfico que gera forte impacto sobre as mudanças climáticas e aumenta a escassez da água.

Palavras chaves: Gestão Integrada. Recursos hídricos. Governança. Água.

\section{INTRODUCCIÓN}

Durante toda la existencia de nuestra vida humana necesitamos y dependemos del uso del agua, para nuestra subsistencia, por ello existe la imposibilidad de entender la vida en nuestro mundo sin agua. Las comunidades siempre se han asentado y desarrollado cerca de las fuentes de agua o ríos, permitiendo el trabajo de las tierras para cultivar sus alimentos a través de actividades agrarias logrando convertirse en sedentarios (Burstein-Roda, 2018). Como se ve, el comportamiento de la sociedad siempre ha seguido este camino, con el objetivo de un rápido y mejor acceso al recurso.

Por otro lado, nuestro planeta está cubierta hasta el $70 \%$ por agua y el resto es tierra firme, sin embargo, el agua que está apta para consumo humano es mínima, por lo que se estima que sólo el $0.77 \%$ es agua dulce y se encuentra accesible al ser humano; por lo que es posible decir que el agua es un recurso finito, escaso y vulnerable (CONAGUA, 2011). En esa misma línea, la gestión de los recursos hídricos se vuelve un desafío para el estado y la sociedad, por lo que el cuidado y la gestión del agua como recurso natural aumenta a medida que pasa el tiempo, debido a que la sociedad y las ciudades están en constante crecimiento, mientras que el acceso al recurso agua es más complejo y difícil (Cairampoma, 2019). Por ello, el reto está en buscar una solución al problema de la escasez del agua.

Del mismo modo, se proyecta un incremento de la demanda mundial del nivel actual de uso del agua del $20 \%$ al $30 \%$ para el 2050. Asimismo, más de 2 mil millones de habitantes en el planeta sufren una fuerte escasez de agua, y aproximadamente 4 mil millones la adolecen por lo menos un mes al año (Naciones Unidas, 2019). Evidentemente, estos indicadores debemos tenerlos presentes para la toma de decisiones oportuna, antes que sea demasiado tarde. Es preciso indicar que, la proyección al año 2025 realizada por la Organización Mundial de la Salud indica que, la mitad de la población mundial habitará en lugares con escasez de agua, es por ello que se debe garantizar la disponibilidad de agua y su gestión sostenible (Defensoría del Pueblo, 2019). Ante esta situación, la articulación vertical y horizontal debe buscar los mecanismos de asegurar el balance y la disponibilidad hídrica. 
Asimismo, en el Perú existe la creencia de que en la selva amazónica existe una gran cantidad de agua y por consiguiente nos sobra, cuando en realidad eso solamente sucede en las ríos, porque el recurso hídrico es insuficiente para el desarrollo de la agricultura en las chacras de cultivo y sobre todo en los hogares; a esto se suma, la deficiente infraestructura, frágil compromiso de los usuarios o baja participación de las mujeres en las organizaciones, los cuales son algunos problemas que perjudican la buena gestión de los recursos hídricos (ANA, 2019). Por lo tanto, las Autoridades del Agua muestran deficiencias en el cumplimiento de sus funciones, debido a la limitada articulación con los gobiernos regionales y locales, a esto se suma la indiferencia de nuestros funcionarios, débil voluntad política por parte de nuestros gobernantes y casi nula participación de la sociedad civil que por falta de información no se involucra en la gestión de los recursos hídricos.

\section{REVISIÓN DE LA LITERATURA}

De acuerdo con la UNESCO (2009), la Gestión Integrada de los Recursos Hídricos (GIRH) apareció alrededor de la década de los años 80 en reacción a las crecientes presiones sobre los recursos hídricos a causa de la competencia entre distintos usuarios por un recurso limitado, el reconocimiento de las condiciones del ecosistema, la contaminación y el riesgo de disminución de la disponibilidad de agua en virtud del cambio climático. Asimismo, la GIRH, está definido como el proceso que está relacionado directamente con la administración, supervisión y control del sistema (UNESCO, 2012). De similar forma, la Global Water Partnership - GWP e International Network of Basin Organizations - INBO (2009) define la GIRH como un proceso que consiste en promover el desarrollo coordinado, la gestión del agua, los recursos asociados y la tierra, a fin de maximizar el resultado del bienestar económico y social con equidad sin poner en riesgo la sostenibilidad de los ecosistemas. Resumidamente, la GIRH es una noción lógica y atractiva, que se basa en que los distintos usos de los recursos hídricos son interdependientes, lo cual implica que se deben de considerar en forma conjunta (Indij, Paris y Schreider, 2015); por consiguiente, según Cap-Net (2005), la elevada demanda de agua para uso en regadíos y flujos de drenaje contaminados de la actividad agrícola, generan una menor cantidad de agua fresca para beber o para uso industrial; las aguas residuales de los gobiernos locales y de la actividad industrial, contaminan los ríos y conminan los ecosistemas.

Asimismo, el calentamiento global, deforestación, agricultura, minería y otros; son factores que afectan los recursos hídricos del planeta; es por ello que, el suministro, la calidad y la gestión del agua deben ser integrales. Dentro de este marco, la UNESCO (2012), conceptualiza a los recursos hídricos, como aquellos recursos que se encuentran o están potencialmente disponibles, en calidad y cantidad suficiente, en un lugar y en un periodo de tiempo dado, destinados para satisfacer una demanda identificable. Se puede señalar que, la GIRH permitirá lograr con éxito los Objetivos de Desarrollo Sostenible $N^{\circ}$ 1, 2, 6, 11, 12,14, 16 y 17 (UN, 2020). Por otro lado, trabajar la seguridad hídrica permitirá tener la capacidad de dar agua en cantidad y calidad suficiente y satisfacer necesidades domésticas, agrícolas, industriales y de energía, por eso Cassin y Locatelli (2020) proponen a manera de definición de la seguridad hídrica, como: i) la disponibilidad de agua que sea apropiada en calidad y cantidad para el suministro humano, subsistencia, protección de los ecosistemas y producción; ii) la capacidad institucional, infraestructura y financiera para acceso y beneficio del agua de forma sostenible y emplear la interrelación entre tipos de usos y sectores; y iii) un nivel aceptable de riesgos para la sociedad, economía y ambiente que se asocien a recursos hídricos.

Desde esta perspectiva, la GIRH se basa en los principios de Dublín, que se definen de la siguiente manera: 1) El agua dulce es un recurso finito y vulnerable, propio para sostener la vida, desarrollo y medio ambiente; 2) El desarrollo y la gestión del agua deben basarse en un enfoque 
participativo, que involucre usuarios, planificadores y formuladores de políticas a todo nivel; 3) La mujer desempeña un papel fundamental en el suministro, la gestión y la protección del agua; 4) El agua es un bien público y tiene valor social y económico en todos sus usos competitivos y 5) La GIRH se basa en la gestión equitativo y eficiente y el uso sostenible del agua (Villena, 2018). En esa misma línea, los objetivos que persigue la GIRH, de acuerdo a como lo describe Alcácer (2019) son los siguientes: 1) el agua es un recurso para beber y lavarse, pero también se necesita para subsistir, 2) tanto la oferta como la demanda de agua se deben considerar al crear estrategias de gestión, 3) el agua es un bien económico, social y ambiental, 4) la participación de los actores locales debe ser parte de la toma de decisiones sobre sus recursos y 5) reconocer el papel de la mujer en la recolección, distribución y gestión del agua (p. 9). En términos generales, el marco legal y normativo actual, comparado con el escenario anterior, ha tenido un gran avance en donde la GIRH se caracterizaba por el predominio de una visión sectorial y por la dispersión normativa e institucional, lo que nos llevó a un escenario de ingobernabilidad y a la desarticulación de los objetivos de desarrollo sostenible.

De esta manera, la puesta en marcha de la GIRH debe seguir el Manual propuesto por la Global Water Partnership (GWP) e International Network of Basin Organizations (2009) que facilita un modelo que permite mejorar la gobernabilidad de los recursos de agua dulce, el mismo que describe los aspectos claves que se deben cumplir que son los siguientes: i) creación de sistemas de gestión de cuencas; ii) definir los roles y tipos de organismos de cuenca; iii) financiamiento; iv) participación de las partes interesadas; v) la planificación estratégica a largo plazo; vi) planes de acción a nivel de cuencas; vii) sistemas de información y monitoreo de cuencas y viii) comunicación. Sobre todo, para lograr una gestión conveniente de los recursos hídricos y, por lo tanto, la seguridad del agua, es importante que la gestión tenga la capacidad de responder a muchos procesos, por la cual las tendencias globales en el impacto político, social y económico y su impacto en la región ha identificado fenómenos globales que cambiarán el futuro de América Latina y el Caribe, como son los recursos naturales y los mercados mundiales, las transformaciones demográficas y los ingresos de la personas, y los procesos de urbanización y expansión de las ciudades (Peña, 2016).

Por ello, la GIRH es un asunto muy complejo, ya que es un proceso constante y de largo plazo, para lo cual se requiere el aporte e interrelación de gobiernos, agencias y organizaciones a niveles internacional, nacional, regional y local, del sector privado, así como de organizaciones benéficas y de los individuos dedicados (ONU Medio Ambiente, 2018). Además, tal como lo sostienen Saravanan, McDonald y Mollinga (2009), el enfoque de la GIRH está sujeto fundamentalmente a la organización de las instituciones y la normativa; por lo que todos los actores se relacionan utilizando reglas y recursos en distintos niveles, espacios y tiempos. Por otro lado, desde la óptica socioecosistémica, Maass (2015) define a la cuenca como un sistema complejo humano-bio-físico integrado, ya que sus elementos hacen que el comportamiento del agua no sólo sea producto de procesos naturales y leyes, sino también de procesos sociales. Cabe destacar lo que menciona Paris y Mariano (2017), que la cuenca hidrográfica es una unidad territorial muy complicada, la misma que sobreviene no sólo de las peculiaridades naturales, sino de las singularidades de las personas y por la dinámica que proporciona la transformación histórica de este territorio.

\section{PROCEDIMIENTOS METODOLÓGICOS}

Se pasa a detallar los procedimientos seguidos para la realización de la presente investigación: 


\subsection{El diseño y el sitio del estudio}

Se trata de una exploración sistemática, desarrollado por profesionales doctorandos en Gestión Pública y Gobernabilidad, de la Escuela de Posgrado de la Universidad César Vallejo, Perú. La obtención de datos fue realizada en cuatro meses. Para realizar esta revisión, sobre procesos de implementación de la GIRH en nuestro planeta, se tomaron artículos científicos de los distintos continentes del planeta, de los cuales 52\% corresponde a América del Sur, 24\% a América del Norte, el $9 \%$ a Asia y el resto $15 \%$ al resto de continentes.

\subsection{Criterios de Selección}

Tipos de intervinientes: se incorporaron estudios que implicaban a adultos, personas mayores dentro de la comunidad general y literatura de ámbito teórico. Tipos de estudios: Teniendo en cuenta el número amplio de estudios sobre recursos hídricos en el planeta, el objetivo de esta breve reseña es trazar un mapa de los conocimientos existentes sobre el tema e identificar los diseños de estudio por el nivel de las pruebas. Tipos de resultados: Se eligieron como resultados primarios a: publicaciones originales de estudios científicos e información reciente. Búsqueda de estudios: Se buscó manualmente utilizando las referencias de los estudios primarios y secundarios encontrados en la búsqueda electrónica. La indagación se realizó directamente en la base de datos EBSCO, Scopus, Science Direct, SciELO, ProQuest y Redalyc, para lo cual se utilizó el filtrado por Publicaciones Arbitradas y Académicas por el periodo del 2016 al 2020. Selección de estudios: El proceso de elección de los estudios fue realizado por dos revisores independientes, y cualquier divergencia fue resuelta por un tercer revisor. Los artículos científicos se seleccionaron en dos etapas. El primer paso consistió en revisar los títulos y resúmenes de los artículos encontrados con nuestra estrategia de búsqueda; se seleccionaron los estudios potencialmente elegibles. El segundo paso consistió en revisar el escrito completo de los artículos preseleccionados para confirmar su elegibilidad.

\subsection{Análisis de datos}

La estrategia de la indagación dio como resultado 1801 referencias. En este proceso de elección se descartaron 1675 referencias que no satisfacían los criterios de inclusión tras verificar el título y el resumen. Las 126 citas seleccionadas fueron leídas para convalidar la selección. Una vez finalizado el proceso de aceptabilidad, se incluyeron treinta y tres (33) artículos científicos. La revisión de las 126 citas de texto completo seleccionadas resultó en el descarte de 93 estudios que no cumplían con nuestros criterios de inclusión. Los resultados de los 33 artículos restantes se presentan de manera sistemática y descriptiva, ya que la naturaleza de estos estudios no permite ningún otro tipo de análisis de igual manera.

\section{RESULTADOS}

La investigación reveló muchas coincidencias sobre la implementación del enfoque de la Gestión Integrada de los Recursos Hídricos (GIRH) en el planeta, los mismo que concuerdan sobre la importancia del cuidado de agua, ya que es el medio de vida de las personas, sin ella no habría agricultura para producir alimentos, lo mismo que genera mejores condiciones económicas y sin dejar de lado la sostenibilidad ambiental. De acuerdo con Gil y Reyes (2015), la GIRH se fundamente en tres dimensiones o pilares que son equidad social, eficiencia económica y sostenibilidad ambiental; y para lograr su implementación se necesitan herramientas de gestión que nos permitan evaluarla, para ello, es necesario un ambiente propicio que permita fijar políticas y normas, además de un marco institucional a distintos niveles. 
Debido a la escasez del agua cada vez más crítica y la necesidad de mantener el balance hídrico, Madrigal et al. (2020), sostienen que las personas están dispuestas a pagar más por el servicio o suministro del agua que utilizan. Por otro lado, la deficiente e insuficiente recaudación por tarifas de agua, genera problemas para cubrir gastos de estudios, programas, proyectos y obras relacionados al recurso hídrico en la cuenca (Gomes, Meiguins y Martins, 2020). De la misma forma, se afirma que sin agua no hay agricultura, por eso Mekonnen, Abeje y Addisu (2020), sostienen que trabajar en la conservación del recurso hídrico influencia en el rendimiento de la producción agrícola; además, Vallino, Ridolfi y Laio (2020) validan la información, indicando la existencia de una relación entre los avances de los niveles de la GIRH y producción agrícola, generando un aumento en el rendimiento de hasta un 13\%. En esa misma línea, Zhang et al. (2020) afirman que la menor eficiencia en el consumo del agua en la agricultura genera alto impacto en el índice de seguridad hídrica (D’Ambrosio et al, 2020).

Asimismo, Tsani, Koudouri y Akinsete (2020) refieren que se están dando pasos valiosos en la consolidación de la GIRH, para ello es necesario perfeccionar el diseño y la implementación de las políticas. Además, Cano y Haller (2018) afirman que, en los Consejos de Cuenca los Gobiernos Regionales ocupan la presidencia por su vinculación con el territorio, sin embargo, su representante no tiene poder de decisión. Por otra parte, Dávila y Covarrubias (2017) afirman que la GIRH adopta una política más inclinada hacia los objetivos económicos; de modo idéntico, Urquiza y Billi (2020) aseveran que, las organizaciones de usuarios dirigen en mayor parte su atención a la dimensión económica. Cabe destacar que, la sostenibilidad de recursos hídricos y los medios de vida rurales en áreas protegidas o de conservación, se encuentran en una situación de pobreza generalizada, tal como lo señalan Caro-Carrero, Carmona-Jiménez y Figueroa (2020), por lo que su objetivo es la supervivencia y a esto se suma el mal planteamiento de la regulación en el uso y la gestión del agua, además, afirman que el $68 \%$ de los alimentos que se consumen provienen de los pequeños agricultores y que el $62 \%$ de los ciudadanos de dichas áreas protegidas dependen del agua para su sustento.

Las personas necesitan el agua para su subsistencia, es por ello que tienen la prioridad de su uso para consumo. En relación a ello, Madrigal et al. (2020) evidencian que, ante la escasez de agua, algunas personas se mostraron menos dispuestas a facilitar la distribución equitativa del líquido vital, a pesar que el $98 \%$ de las personas encuestadas consideró que el agua es de todos, el 93\% de las personas considera que estaría dispuesta a compartir el recurso ante una posible escasez y el $88 \%$ considera que el recurso agua es agotable y son conscientes de su vulnerabilidad. Asimismo, D’Ambrosio et al. (2020) manifiestan que las actividades de la ciudadanía dañan las cuencas, requiriendo la rehabilitación de las aguas superficiales, subterráneas, suelo y sub suelo.

Sin duda alguna, si no hay planificación y articulación de los actores de la cuenca no se logrará la implementación de la GIRH, por ello Mendoça, Marini y Mariko (2020), afirman que la articulación vertical de la planificación y GIRH supone la adecuación de las demandas identificadas de las cuencas y sub cuencas; y la articulación horizontal debe poner en acción a todos los actores del sistema para que sea más eficiente y de uso racional. Por su parte, Urquiza y Billi (2020) aseguran que la comunidad siente que las organizaciones de usuarios les han dado la espalda, desarrollando sus propias regulaciones por lo que son muy criticadas. Es importante agregar, que la participación de los actores civiles locales es muy limitada, tal como lo atestiguan Mancilla y Bodin (2019), donde manifiestan que las partes interesadas no expresan sus ideas en el marco de discursos de expertos, por lo que a menudo son marginados y no son escuchados. En consecuencia, la información y el conocimiento de los diferentes actores debe ayudar a mejorar las prácticas de los usuarios del agua para la asignación de recursos limitados, generando el marco normativo 
actualizado para que sea más efectiva y justa, tal como lo manifiestan Prakongsri y Santiboon (2020); por ese motivo para Agramont et al. (2019) las políticas nacionales no consideran el conocimiento o experiencia local como factor importante en la solución sostenible de los recursos hídricos.

De acuerdo con Yahn (2020), afirma que el proceso de gobernanza lleva a la implementación de la GIRH, por ello es importante incluir a los actores subnacionales en las negociaciones y a otros actores como las tribus nativas americanas, que ancestralmente han ocupado la cuenca, por consiguiente, Manzano-Solís et al. (2019) manifiesta que para lograr la implementación de la GIRH es importante la identificación de los interesados que puedan mediar en las variables claves identificadas, dentro del contexto de la gobernanza del agua. En este mismo contexto, Lizcano et al. (2019) reconocen los derechos de la comunidades indígenas y pueblos para usar sus costumbres y procedimientos ancestrales en la preservación de los recursos hídricos.

De igual forma, Salmoral et al. (2020) manifiestan que en Arequipa - Perú existe una red compleja de actores involucrados del agua para la gobernanza del desarrollo sostenible, donde la participación exitosa podría promoverse a través de instrumentos formales e informales, que incluyan políticas e iniciativas ejemplares entre sectores y actores, sin embargo, se encontraron visiones compartidas y prioridades contradictorias relacionadas con la gestión sostenible del agua. En ese mismo orden de ideas, Cano y Haller (2018) evidenciaron una falta de visión política para la planificación integral del agua. Asimismo, Antón-Chávez y García-Yovera (2019) manifiestan que en Perú la participación de la mujer es muy limitada llegando a sólo 14.4\%, además de la alta ausencia en los procesos de capacitación, el mismo que llega a $69.8 \%$, identificando como factores la lejanía y los horarios inadecuados. Por otra parte, Brandshaug (2019) sostiene que la gestión del agua debe ser abordada no sólo como un bien económico sino también como un bien común.

Por otra parte, Libanio (2018) da a entender que la implementación de la Política Nacional de Recursos Hídricos en Brasil, tiene como iniciativa la inclusión de actores no estatales en las decisiones públicas, esto no se traduce necesariamente en el empoderamiento de los grupos sociales, ni garantiza una mayor democracia y eficiente proceso de gobernanza. Asimismo, Lerner et al. (2018) revelan que el marco del problema del agua es el crecimiento urbano y trae como consecuencia una mayor demanda, generando impactos en la infraestructura, por lo que esta presión demográfica se asocia con una mayor frecuencia de escasez, la cual es avalada por la normativa de aguas que indica que todos los residentes tienen derecho al agua, presionando al estado por servicios públicos que intercambian por votos.

El agua como parte del medio ambiente cada vez se ve más perjudicado por la presión que producen la personas sobre ella, ya que necesitan para su desarrollo y subsistencia. Ante ese escenario, Urquiza y Billi (2020) afirma que en Chile los gestores del agua no le dan la debida importancia a la dimensión ambiental. Por su parte, Gomes et al. (2020) manifiestan que la aplicación en Brasil de una misma Política Ambiental Nacional, genera que los indicadores de gestión de los todos los municipios de la cuenca sean similares en cuanto a cómo realizan la gestión ambiental, a esto se suma el limitado personal profesional. Por otro lado, las actividades antropogénicas que generan estrés hídrico, también producen el calentamiento global, por ello Mekonnen et al. (2020) afirman que el 93\% de los entrevistados percibieron la existencia de cambio climático. 
Asimismo, los desafíos que enfrenta el mundo con respecto a la conservación de las cuencas son muy grandes, en función a ello D'Ambrosio et al. (2020) aseveran que la evaluación de sostenibilidad mostró que la contaminación era el factor principal que impacta en la seguridad del agua. De la misma forma, la gestión del agua no es proceso estático, más bien es muy dinámico, por ello Tsani et al. (2020) expresa que es necesario perfeccionar el diseño y la implementación de las políticas, las cuales deben alinearse hacia los objetivos de desarrollo sostenible, apuntando convenios ambientales y escenarios de cambio climático. En esa misma línea, es inminente que la GIRH está globalizada, por eso Cáñez (2018) afirma que la influencia internacional impacta en la agenda gubernamental y se debe tomar distintos compromisos internacionales como los Objetivos de Desarrollo del Milenio, los Objetivos de Desarrollo Sostenible, además, de las estrategias del cambio climático y la GIRH. Se puede señalar que, en el proceso de la GIRH se presentarán distintas discrepancias sobre la implementación, sobre esto Castro, Vélez y Madrigal (2018) revelan la existencia de un complejo contexto socio ambiental que se incrementa, cuando se trata de regular el agua y se encuentra dispersa en varios ámbitos normativos, como el referido al ambiente y a los servicios públicos; cuyos fines discrepan, por un lado, la protección de la naturaleza y por otro la eficiencia de los servicios públicos.

De acuerdo a lo citado al inicio de los resultados, las dimensiones se operativizan con herramientas de gestión que nos permitan hacer seguimiento y evaluación, por ello He et al. (2020) compararon la gestión de los recursos hídricos entre China y Estados Unidos, dando como resultado la necesidad urgente de nuevos paradigmas de armonía entre las personas y el agua, el empoderamiento de las comisiones de las cuencas con autoridad integral y el uso del agua para los servicios del ecosistema que debe ser una parte de la GIRH. Por su parte, las Instituciones públicas son los organismos responsables de llevar a cabo los procesos para la implementación de la GIRH, para ello deben articular con los demás actores de la cuenca, sin embargo, esta función se desarrolla de manera muy deficiente, debido a ello Urquiza y Billi (2020) afirman que la capacidad de las instituciones estatales para gestionar el acceso a los recursos hídricos es ampliamente cuestionada. En relación a lo referido, Gomes et al. (2020) señalan que los municipios carecen de suficiente personal técnico, lo que dificulta las tareas de otorgamiento de licencias y falta de registro en el Sistema Nacional de Información.

Ahora bien, los países tienen diferentes estrategias para implementar la GIRH, esto depende de la cultura de su población, capacidad técnica y sistema político, es por ello que Miranda y Reynard (2020), señalan que en Brasil los Comités de Cuenca son responsables de implementar las herramientas de gestión del agua proporcionadas por la ley nacional, en cambio en Suiza es flexible a la hora de elegir su modelo de gestión del agua, depende de los intereses locales; además de lo señalado, Libanio (2018) demuestra que los agentes gubernamentales no valoran los espacios públicos promovidos por el Sistema Nacional de Gestión de los Recursos Hídricos de Brasil, como ventanas de oportunidad para acercar los temas a la agenda de decisiones del sector del agua. Por su parte, Kauffer y Mejía (2020) manifiestan que la implementación de la política hídrica en Belice no se terminó de consolidar, generando un cambio institucional, por lo que más que la aprobación de una ley y la creación de la Autoridad del Agua se requiere cambios adicionales, normativos y en el aparato institucional, que se realizaran dentro de los objetivos de la GIRH. Asimismo, PachecoVega (2020) analiza el aparato institucional y el marco legal de la gobernanza del agua, lo cual arroja resultados muy importantes relacionado a las dificultades institucionales y desajuste jurisdiccional, a pesar que la GIRH se enmarca en el principio de que mejora la participación de los actores en la gobernanza del agua. 
Cabe indicar que, en Chile las organizaciones de usuarios son los que regulan el agua, generando una fragmentación debido a que dan mayor atención a los grandes agricultores y dan la espalda a los pequeños, ante esta situación Rojas (2020) da a entender que la implementación de la GIRH en Chile es posible bajo el actual Código de Aguas, si bien no hace referencias explícitas, pero pueden verse indicios de ello implícitamente; sólo sería necesario otras disposiciones de carácter reglamentario. Por el contrario, como la manifiesta Silva, Herreros y Borges (2017) los resultados muestran que Brasil ha dado pasos importantes y ha avanzado en términos de gestión, derechos de agua, uso y se ha ido incorporando paulatinamente al modelo de la GIRH.

Del mismo modo, Pulgarín y Mejía (2017) manifiestan que las capacidades administrativas, económicas y financieras de los gobiernos locales en Colombia, necesitan de los recursos suficientes para llevar a cabo la implementación en la GIRH. Por otra parte, el Perú no es ajeno a las deficiencias en la implementación de la GIRH, por ello French (2016) da a conocer que la burocracia hídrica en la actualidad ha consolidado una autoridad centralizada sobre el agua mediante la asimilación y desarrolló una variante de la GIRH que prioriza el establecimiento de derechos formales de agua, el reconocimiento del valor económico del recurso hídrico y un creciente enfoque en la eficiencia del uso del agua. En consecuencia, Alcácer (2019), refiere que los objetivos de la GIRH son muy ambiciosas y tiene como hipótesis la mejora significativa en las tres dimensiones, social, económica y ambiental; el mismo que no se sostiene en la evaluación empírica y de demostración científica, con los cuales se pueda estimar el logro de los principios que establece; es por ello, que a lo largo de la experiencia de más de tres décadas, es necesario plantear una mejoría abordando tres elementos: primero, abandonar ese concepto idealizado para aceptar que la GIRH no es infalible; segundo, flexibilizar el grado de trabajo, trasladando el foco del agua como recurso al agua como conflicto y tercero, aceptar la falta de conocimiento y mejora en la gestión, abordando las evaluaciones de su implementación desde un punto de vista científico.

\section{DISCUSIÓN}

La GIRH se sostiene en los pilares de eficiencia económica, equidad social y sostenibilidad ambiental. Sin embargo, no debemos dejar de lado la institucionalidad como medio eficiente para lograr la consolidación de la GIRH.

\subsection{Económico}

La gestión del agua nos va a permitir asegurar el futuro de nuestra existencia y para ello es importante la existencia de una regulación eficiente por parte del estado, lo cual es vital para la consolidación de la GIRH, es por este motivo que no debemos enfocarnos solo en el pilar económico, sino también en el tema social, cerrando brechas en infraestructura y saneamiento, y en lo ambiental protegiendo las cabeceras de cuenca, para ello la inversión pública y privada debe desarrollar proyectos de reforestación con participación de los actores civiles directos e indirectos asentados en el dicho territorio. Ante esta realidad, la pandemia mundial del Covid-19 nos ha mostrado la importancia de los alimentos producidos por los agricultores y el estado debe cumplir con su rol de promotor de actividades productivas que generen cadenas de valor para maximizar sus beneficios y mejorar la calidad de vida de las personas. Por ello se puede afirmar que, sin agua no existe desarrollo económico, lo cual genera puestos de trabajo, energía, turismo, agricultura sostenible y mejores condiciones de vida. Asimismo, los recursos económicos que recaudan las Juntas de Usuarios son insuficientes que nos les permite mantenerse como institución, a este se suma el limitado presupuesto público que asigna el estado en los distintos niveles de gobierno para el desarrollo de proyectos relacionados a la sostenibilidad del recurso hídrico. 


\subsection{Social}

Es común percibir que, las personas no se preocupan por que existe una aparente abundancia de agua en nuestro planeta, mucho más en el continente americano, pero esto no es del todo cierto, por lo que tenemos tomar acciones inmediatas como actores directos, ya que la escasez del recurso es un potencial generador de conflictos, a esto se suma el crecimiento urbano que produce una mayor demanda de agua y cambio climático, generando mayor presión al estado para brindar mayores servicios públicos a cambio de votos. De la misma manera, las actividades antropogénicas son los mayores causantes de estrés hídrico; por consiguiente, la articulación vertical ayudará a promover la planificación en los distintos niveles de gobierno y la articulación horizontal de los actores civiles generará conciencia y cambio de actitud, que conllevará a una mayor eficiencia y uso racional del recurso hídrico. Por el contrario, la existencia de una limitada participación de los actores civiles trae como consecuencia no tomar en cuenta sus ideas; asimismo los expertos en temas de gestión del agua no consideran sus opiniones produciendo aún más ese distanciamiento entre estos los actores; debido a ello se pierde los conocimientos o experiencias locales para la solución de los problemas de los recursos hídricos; siendo esto una realidad latente en la gestión de la cuenca.

En esa misma línea, los gestores del agua tienen una limitada consideración a los derechos ancestrales para la preservación de los recursos, a esto se suma la casi nula participación de la mujer en los procesos de gestión, dejando de lado el enfoque intercultural y el enfoque de género. Por otra parte, los Consejos de Cuenca son espacios de concertación que su consolidación tienen muchas dificultades, por lo que deben ser fortalecidos ya que demuestran una grave falta de coordinación y visión política. Sin duda alguna, los gestores del agua no dan la debida importancia a la sostenibilidad ambiental y si no tomamos acciones inmediatas estaríamos hipotecando el futuro de las nuevas generaciones, por ello en la actualidad la percepción de la sociedad evidencia el cambio climático, lo cual se ve reflejado en los periodos largos de escasez de agua y precipitaciones continuas que producen inundaciones y ponen en riesgo la vida humana, llegando muchas veces a la muerte. Para ello, es importante promover los compromisos internacionales dentro de ellos los Objetivos de Desarrollo Sostenible y la GIRH.

\subsection{Ambiental}

La investigación ha evidenciado que el modelo de la GIRH aplicada en Chile, nos demuestra que no existe articulación entre los tres pilares, económico, social y ambiental y si esto no se concibe como tal podría generar consecuencias imprevisibles y desafíos aún más profundos. A este se suma la existencia de un estado debilitado que no cumple su rol regulador, siendo las empresas privadas y las Juntas de Usuarios dueños del manejo del agua, generando desigualdad en la asignación del recurso. Ante esta situación, la política ambiental en el planeta debe adecuarse a la realidad de cada cuenca, tomando en cuenta el nivel socio cultural y económico de la sociedad, lo mismo que permitirá obtener mejores resultados en la GIRH. La sostenibilidad ambiental no cuenta con la debida importancia, debido a ello la asignación de recursos humanos para las actividades relacionadas es limitado, no contando con los profesionales suficientes para las inspecciones, supervisión y fiscalización. Por lo descrito, se puede afirmar que tenemos mucho que aprender, que la protección del medio ambiente no signifique que no podemos tocar, sino debemos buscar las estrategias para el uso del recurso agua de manera sostenible y las normas deben estar adecuadas a cada realidad, caso contrario en el futuro la raza humana estaría destinado a su extinción. 


\subsection{Institucionalidad}

Por su parte, la institucionalidad de la gestión de los recursos hídricos necesita urgentemente nuevas formas en los procesos de implementación, con nuevos paradigmas, en armonía entre las personas y los recursos hídricos, para ello las instituciones públicas son responsables de articular con todos los actores de la cuenca promoviendo el enfoque intercultural y de género; tomando en cuenta la cultura del ciudadano, la capacidad técnica y el sistema político de una cuenca, existiendo una flexibilidad en la elección de un modelo de gestión del agua que dependerá de los intereses locales. Cabe mencionar que, los gobiernos locales no cuentan con los recursos suficientes para la implementación de la GIRH, por lo que es imposible que se desarrollen las tareas contempladas en los instrumentos de gestión, a esto se suma la consolidación de la burocracia hídrica que ha consolidado el centralismo de la gestión agua.

\section{CONCLUSIONES}

A partir de lo descrito y teniendo en cuenta los pilares de la GIRH, económico, social y ambiental, podemos concluir que la mejora de la gestión del agua permitirá el incremento de la productividad, por consiguiente, va a generar mejores condiciones de vida y oportunidades para los ciudadanos asentados en la cuenca. Por otro lado, los gobiernos subnacionales como dueños del territorio, son los presidentes de los Consejos de Cuenca, por consiguiente, deben cumplir con su rol, designando a sus representantes con poder de decisión para la consolidación de la GIRH. A esto debe sumarse la importancia de la implementación de un mecanismo de pago por servicios ecosistémicos por el uso del agua, además de fortalecer las capacidades a las Juntas de Usuarios para mejorar la recaudación por tarifas de agua y retribución económica, ya que son ellos los que cumplen la función de distribución del agua de manera eficiente, lo cual permite que los agricultores puedan hacer producir la tierra y los alimentos para el mundo.

En ese mismo orden de ideas, la implementación de la GIRH está aún en proceso de conocimiento de los actores que participan en la cuenca, para ello se debe cambiar el enfoque de la intervención, no sólo centrarse en trabajar en el otorgamiento de derechos y licencias, sino trabajar en la articulación vertical y horizontal bajo el enfoque de gobernanza. Por otro lado, la deficiencia en la implementación se muestra más acentuada en países latinoamericanos, por lo que se debe generar políticas y normas regulatorias que conciban la experiencia y el conocimiento de los actores asentados en la cuenca, con enfoque intercultural y de género; con la participación activa de la mujer en la gestión. Definitivamente, se propone mejoras a la GIRH, lo cual debe abordar algunos elementos adicionales, como el desprendimiento de la idealización aceptando que la GIRH no es infalible; además de enfocarnos en el agua como recurso y aceptar la necesidad de fortalecimiento de capacidades, sensibilización y mejora en la gestión, concibiendo a la investigación científica dentro de las evaluaciones de su implementación.

Es preciso señalar que existen algunas limitaciones en la investigación, que pueden ser utilizadas para futuros estudios. La primera, referida a que la mayoría de artículos científicos revisados han sido realizados desde el punto de vista tecnocrático y muy poco, sobre el proceso de gestión de los recursos hídricos, ni participación de actores directos, que generación tras generación ocupan las cuencas; para ello se puede desarrollar estudios empíricos que nos ayuden a comprender la realidad, el pensamiento, la idiosincrasia, los modos de vida y la concepción que tienen estos actores sobre cómo gestionar el agua. La segunda limitación se relaciona con la selección de artículos científicos enfocados sólo al tema ambiental, pudiéndose explorar otros campos como los sociales, que permitan confirmar que la implementación del enfoque es multidisciplinaria. Y como tercera, fue la selección de artículos según zona geográfica, con inclinación al continente americano por residir en ella, encontrándose predominantemente estudios empíricos y teóricos; por lo que sería 
sumamente importante incorporar estudios del continente europeo, que por su alta tecnología y características propias podrían fortalecer la gestión con nuevos paradigmas que articule a los actores directos e indirectos.

Finalmente, como recomendación es necesario que la gestión del territorio se desarrolle bajo el enfoque de cuenca, debiendo ser liderado por los Gobiernos Subnacionales, para ello se debe trabajar a través de grupos especializados responsables de articular a los demás actores, generando mecanismos de motivación para tener encendida la mecha de la Gestión integrada de los Recursos Hídricos.

\section{REFERENCIAS}

Agramont, A., Craps, M., Balderrama, M. \& Huysmans, M. (2019). Transdisciplinary learning communities to involve vulnerable social groups in solving complex water-related problems in Bolivia. Water. 11(2), 385. https://doi.org/10.3390/w11020385.

Alcácer, C. (2019). Paradigmas de la gestión integrada del agua (I): Una crítica evolutiva a la gestión integrada de los recursos hídricos(GIRH). Revista Científica ECOCIENCIA, 6(2), 1-21. https://doi.org/10.21855/ecociencia.62.164.

Autoridad Nacional del Agua - ANA (mayo 2019). La batalla por el agua se juega en la cancha. Recuperado de https://repositorio.ana.gob.pe/handle/20.500.12543/3642.

Antón-Chávez, A. y García-Yovera, S. (2019). Comunicación interna en organizaciones agrarias administradoras de agua, Perú. Revista Venezolana de Gerencia. 24(2), 557-572. http://dx.doi.org/10.37960/revista.v24i2.31510.

Área del Medio Ambiente: Adjuntía del Medio Ambiente, Servicios Públicos y Pueblos Indígenas (2019). Defensoría del Pueblo. Recuperado de https://www.gob.pe/institucion/defensoria-del-pueblo/informesublicaciones/1050571-serie-informes-de-adjuntia-01-2019-dpamasppi-ma.

Burstein-Roda, T. (2018). Reflexiones sobre la gestión de los recursos hídricos y la salud pública en el Perú. Revista Peruana de Medicina Experimental y Salud Pública. 35(2), 297-303. https://doi.org/10.17843/rpmesp.2018.352.3641.

Brandshaug, M. (2019). Water as more than commons or commodity: Understanding water management practices in yanque, Perú. Water Alternatives. 12(2), 538-553.

Cano, D. y Haller, A. (2018). Los servicios ecosistémicos hidrológicos: entre la urbanización y el cambio climático. Percepción campesina y experta en la sub cuenca del río Shullcas, Perú. Espacio y Desarrollo. 31, 7-32. https://doi.org/10.18800/espacioydesarrollo.201801.001.

Cáñez, A. (2018). La influencia de las estructuras de gobernanza internacional en la política hídrica mexicana. Norteamérica, Revista Académica del CISAN-UNAM. 13(2), 85-109. https://doi.org/10.22201/cisan.24487228e.2018.2.334.

Cap-Net. (2005). Planes de gestión integrada del recurso hídrico: Manual de capacitación y guía operacional. Recuperado de https://www.gwp.org/globalassets/global/gwp-cam_files/manual-planes-girh.pdf.

Caro-Borrero, A., Carmona-Jiménez, J. \& Figueroa, F. (2020). Water resources conservation and rural livelihoods in protected areas of central Mexico. Journal of Rural Studies. 78, 12-24. https://doi.org/10.1016/j.jrurstud.2020.05.008.

Cassin, J. y Locatelli, B. (2020). Guía para la Evaluación de Intervenciones en Infraestructura Natural. Perú: Forest Trends Association.

Castro-Buitrago, E., Vélez-Echeverri, J. y Madrigal-Pérez, M. (2018). El derecho humano al agua en Colombia: una mirada desde su reconocimiento jurídico en la gestión de cuencas hidrográficas. Gestión y Ambiente. 21(2),195-206. https://doi.org/10.15446/ga.v21n2.73591

Comisión Nacional del Agua - CONAGUA (2011). Estadísticas del agua en México, edición 2011. México: Secretaría de Medio Ambiente y Recursos Naturales. 
D'Ambrosio, E., Ricci, G., Gentile, F. \& De Girolamo, A. (2020). Using water footprint concepts for water security assessment of a basin under anthropogenic pressures. Science of the Total Environment. 748(141356). https://doi.org/10.1016/j.scitotenv.2020.141356.

Dávila, R. \& Covarrubias, G. (2017). Integrated water resources management and the Mexican prospects. Environmental Earth Sciences. 76(390), 1-12. https://doi.org/10.1007/s12665-017-6633-6

French, A. (2016). ¿Una nueva cultura de agua?: inercia institucional y gestión tecnocrática de los recursos hídricos en el Perú. Anthropologica. 34(37), 61-86. https:/doi.org/10.18800/anthropologica.201602.003.

Gil, M. y Reyes, H. (2015). Gestión integral del agua desde un enfoque social, hacia una economía ecológica. México. Nóesis Revista de Ciencias Sociales y Humanidades. 24(47), 158-174. http://dx.doi.org/10.20983/noesis.2015.1.6.

Gomes, S., Meiguins, A. \& Martins, J. (2020). Indicators of hydrological sustainability, governance and water resource regulation in the Moju river basin (PA) - Eastern Amazonia. 263(110354), 1-10. https://doi.org/10.1016/j.jenvman.2020.110354.

Cairampoma, A. (2019). La calidad del agua para el consumo humano en el ordenamiento jurídico peruano. En A. Guevara, W. Obando y F. Segura (Eds.), La gestión de la calidad del agua en el Perú: Sextas jornadas de derecho de aguas (pp. 212-228). Lima, Perú: Pontificia Universidad Católica del Perú.

He, C., Harden, C. \& Liu, Y. (2020). Comparison of water resources management between China and the United States. Geography and Sustainability. 1(2), 98-108. https://doi.org/10.1016/j.geosus.2020.04.002.

Indij, D., Paris, M. y Schreider, M. (2015). Herramientas para Contribuir a la Gestión Sustentable del Agua en Latinoamérica. 10.2788/824991.

Kauffer, E., y Mejía, L. (2020). De la descolonización a la Gestión Integrada de Recursos Hídricos (GIRH): La política del agua de Belice a través del tiempo (1981-2015). Gestión y Política Pública. 29(1), 37-66. https://doi.org/10.29265/gypp.v29i1.656

Libanio, P. (2018). Two decades of Brazil's participatory model for water resources management: From enthusiasm to frustration. Water International. 43(4), 494-511. https://doi.org/10.1080/02508060.2018.1451695.

Lerner, A., Eakin, H., Tellman, E, Bausch, J. \& Hernández, B. (2018). Governing the gaps in water governance and land-use planning in a megacity: The example of hydrological risk in Mexico City. Cities. 83, 61-70. https://doi.org/10.1016/j.cities.2018.06.009.

Lizcano, C., Molina, T., Chamorro, D. y González, L. (2019). Marco legal y teórico sobre el uso de costumbres y tradiciones de los grupos ancestrales en la preservación de recursos hídricos. Dilemas Contemporáneos: Educación, Política y Valores. 7, 1-19. https://doi.org/10.46377/dilemas.v31i1.1187.

Madrigal, H.; Echevarría, S.; Pizarro, Y.; Alfaro, C.; Jiménez, S.; Centeno, J.; López, N. \& Suárez, A. (2020). What do we Think About Water? Public Perception of the Current Situation of Water Resources in Costa Rica: An Indicator of Water Understanding and Management. Uniciencia. 34(1), 159-188. http://dx.doi.org/10.15359/ru.34-1.10.

Mancilla, M. y Bodin, Ö. (2019). Participatory Water Basin Councils in Peru and Brazil: Expert discourses as means and barriers to inclusion. Global Environmental Change. 55, 139-148. https://doi.org/10.1016/j.gloenvcha.2019.02.005

Manzano-Solís, L., Díaz-Delgado, C., Gómez-Albores, M., Mastachi-Loza, C. \& Soares, D. (2019). Use of structural systems analysis for the integrated water resources management in the Nenetzingo river watershed, Mexico. Land Use Policy. 87(104029), 1-11. https://doi.org/10.1016/j.landusepol.2019.104029.

Maass, J. (2015). El manejo de cuencas desde un enfoque socioecosistémico. Cuencas de México. 1(1), 1-44. Recuperado de https://www.riob.org/sites/default/files/IMG/pdf/cuencas_de_Mexico_web-3.pdf

Mekonnen, M., Abeje T. \& Addisu, S. (2021). Integrated watershed management on soil quality, crop productivity and climate change adaptation, dry highland of Northeast Ethiopia. Agricultural Systems. 186(102964), 1-10. https://doi.org/10.1016/j.agsy.2020.102964

Mendoça, S., Pereira, M., Carminato, G. \& Mariko, H. (2020). River Basin Management Plans and their challenges: the case of the Alto-Tietê River Basin - State of São Paulo, Brazil. Ambiente \& Sociedade. 23, e02342. https://doi.org/10.1590/1809-4422asoc20170234r2vu202011ao.

Miranda, G. \& Reynard, E. (2020). Integrated water resources management in federations: The examples of Brazil and Switzerland. Water. 12(7), 1914. https://doi.org/10.3390/w12071914.

Pacheco-Vega, R. (2020). Governing Urban Water Conflict through Watershed Councils - A Public Policy Analysis Approach and Critique. Water. 16(1), 1849. https://doi.org/10.3390/w12071849. 
Paris, M. y Marano, R. (2017). Pautas para la gestión integrada de los recursos hídricos en los bajos submeridionales (Argentina). FAVE - Ciencias Agrarias. 16(1), 57-65. https://doi.org/10.14409/fa.v16i1.6749.

Prakongsri, P. \& Santiboon, T. (2020). Gestión eficaz de los recursos hídricos para las comunidades de la cuenca del río Chi en Tailandia. Environmental Claims Journal. 32(4), 323-348. https://doi.org/10.1080/10406026.2020.1765529.

Pulgarín, J. y Mejía J. (2017). Política pública para la gestión integral del recurso hídrico en Quindío-Risaralda 20082015. Colombia. Gestión y Ambiente. 20(1), 38-49. https://doi.org/10.15446/ga.v20n1.61337.

Rojas, C. (2020). La gestión integrada de recursos hídricos en la regulación de aguas. Identificación y propuesta de avances, a partir de instrumentos vigentes. Revista de Derecho Administrativo Económico. 30, 141-171. https://doi.org/10.7764/redae.30.6

Salmoral, G., Zegarra, E., Vásquez-Rowe, I, González, F. Del Castillo, L., Rondón, G, Graves, A, Rey, D. \& Knox, J. (2020). Water-related challenges in nexus governance for sustainable development: Insights from the city of Arequipa, Perú. Science of the Total Environment. 747(141114), 1-13. https://doi.org/10.1016/j.scitotenv.2020.141114.

Saravanan, S., McDonald, G., \& Mollinga, P. (2009). Critical review of Integrated Water Resources Management: Moving beyond polarised discourse. Natural Resources Fórum. 33, 76-86. https://doi.org/10.1111/j.14778947.2009.01210.x.

Silva, M., Herreros, M. y Borges, F. (2017). Gestão integrada dos recursos hídricos como política de gerenciamento das águas no brasil. Revista de Administración de UFSM. 10(1), 101-115. https://doi.org/10.5902/1983465913358.

Tsani, S., Koundouri, P. \& Akinsete, E. (2020). Resource management and sustainable development: A review of the European water policies in accordance with the United Nations' Sustainable Development Goals. Europa. Environmental Science \& Policy. 114, 570-579. https://doi.org/10.1016/j.envsci.2020.09.008.

UN Environment (2018). Progress on integrated water resources management. Global baseline for SDG 6 Indicator 6.5.1: degree of IWRM implementation. Recuperado de https://www.unenvironment.org/explore-topics/water.

UN (2019). The Sustainable Development Goals Report. United Nations, New York. Recuperado de http://www.un.org/sustainabledevelopment.

Urquiza, A. \& Billi, M. (2020). Water markets and social-ecological resilience to water stress in the context of climate change: an analysis of the Limarí Basin, Chile. Environment, Development and Sustainability. 22, 1929-1951. https://doi.org/10.1007/s10668-018-0271-3.

Vallino, E., Ridolfi, L. \& Laio, F. (2020). Measuring economic water scarcity in agriculture: a cross-country empirical investigation. Environmental Science \& Policy. 114, 73-85. https://doi.org/10.1016/j.envsci.2020.07.017.

Villena, J. (2018). Calidad del Agua y Desarrollo Sostenible. Revista Peruana de Medicina Experimental y Salud Pública. 35(2), 304-308. https://doi.org/10.17843/rpmesp.2018.352.3719.

WWAP (Programa Mundial de la UNESCO de Evaluación de los Recursos Hídricos) (2009). IWRM Guidelines at River Basin Level Parte I Principles. París, UNESCO. Recuperado de https://unesdoc.unesco.org/ark:/48223/pf0000186417.

WWAP (Programa Mundial de la UNESCO de Evaluación de los Recursos Hídricos) (2019). Informe Mundial de las Naciones Unidas sobre el desarrollo de los recursos hídricos 2019: No dejar a nadie atrás. París, UNESCO. Recuperado de https://unesdoc.unesco.org/ark:/48223/pf0000367304.

Yahn, A. (2020). Multi-level water governance without integrated water resources management (IWRM): cooperation in the Columbia River Basin. Ambiente \& Sociedade. 23, e02632. https://doi.org/10.1590/18094422asoc20180263r2vu202014ao.

Zhang, C., Li, J., Zhou, Z. \& Sun, Y. (2020). Application of ecosystem service flows model in water security assessment: A case study in Weihe River Basin, China. Ecological Indicators. 120(106974), 1-10. https://doi.org/10.1016/j.ecolind.2020.106974. 